TRANSCRIPTION

\section{Promoters dictate termination mode}

Transcription termination, which is thought to occur via different molecular mechanisms, prevents transcription readthrough and ensures accurate regulation of gene expression. Miki et al. now describe experiments in Caenorhabditis elegans showing that, surprisingly, the mode of transcription termination is determined by the gene promoter.

In the allosteric model of termination, the polyadenylation (polyA) signal and/or termination signals downstream of the polyA signal ultimately lead to transcription elongation complex (TEC) disassembly. In the kinetic or 'torpedo' model, after cleavage of the pre-mRNA from the TEC, the rest of the nascent transcript that has been synthesized by the TEC is degraded by 5' -3 ' exoribonuclease 2 (XRN2; Rat1 in yeast); the exoribonuclease eventually catches up with the TEC and displaces it from the DNA template. Here, the researchers compared the transcriptome of wild-type nematodes to that of nematodes in which XRN-2 was inactivated by RNA interference or conditional mutations. In the absence of XRN-2, transcription readthrough into downstream regions (up to $50 \mathrm{~kb}$ ) was only detected for a subset of genes, which the authors termed XRN-2-dependent for termination (XDT) genes. Interestingly, the dependence on XRN-2 for termination was not caused by the selective recruitment of XRN-2 to XDT genes, as XRN-2 bound to both XDT and non-XDT genes at transcription end sites in wild-type nematodes.

To map the sequence elements that control which termination mode is used by individual genes in vivo, the researchers expressed a bicistronic reporter construct in nematodes, which requires readthrough from an upstream gene for expression of the downstream reporter (GFP) to occur. They combined promoters, gene bodies and regions downstream of the polyA signal from XDT and non-XDT genes in different permutations to identify determinants of termination mode. Surprisingly, they found that the promoter determines whether transcription termination requires XRN-2. Non-XDT genes possess a sequence element in the region downstream of the polyA signal that is required for transcription termination; that is, termination at non-XDT genes requires compatibility between the promoter and the region downstream of the polyA signal. It will be interesting to dissect how the promoter communicates with the termination machinery, and why XRN-2 is recruited to non-XDT genes but cannot terminate their transcription.

\section{Grant Otto}

Surprisingly, ... the promoter determines whether transcription termination requires $\mathrm{XRN}-2$ 